\title{
NEURAL NETWORK TOWARDS BUSINESS FORECASTING
}

\author{
Prof. A. Maithili ${ }^{1} \quad$ Dr. R. Vasantha Kumari ${ }^{2} \quad$ Mr. S. Rajamanickam ${ }^{3}$ \\ *(Director, Master of Computer Applications, Pope John Paul II College of Education, Puducherry, India) \\ ** (Principal, KWC, Puducherry, India) \\ *** (Research Scholar, Pope John Paul II College of Education, Puducherry, India)
}

\begin{abstract}
It is proposed to have study on a business forecasting based on Neural Networks. The neural networks exhibit mapping capability, they can map input pattern to their associated output patterns. Neural network architectures can be trained with known examples of a problem before they are tested for their inference capacity on unknown instances of the problem. The present paper focuses on Neural Network towards Business Forecasting as well as various factors which influence business forecasting. A special work is carried out towards the implementation of Neural Network at different departments of management, such as Marketing Management, Financial Management, Operational Management, and Risk Management.
\end{abstract}

Keywords - Neural Networks - Artificial Neural Networks - Business forecasting strategy - Influencing factors - Marketing neural forecasting - Financial neural forecasting - Operational neural forecasting - Risk assessment neural forecasting.

\section{INTRODUCTION}

The term neural network traditionally refers to a network of biological neurons. The modern usage of the term refers to artificial neural networks, which are composed of artificial neurons. Thus the term has two distinct usages, Biological neural networks and Artificial Neural Networks. Biological neural networks are made up of real biological neurons that are connected in the peripheral nervous system or central nervous system. In the field of neuroscience, they are identified as a group of neurons that performs a specific physiological function in the laboratory analysis. Artificial neural networks are composed of interconnecting artificial neurons. This network may either be used to gain an understanding of biological neural networks, or for solving artificial intelligence problems.

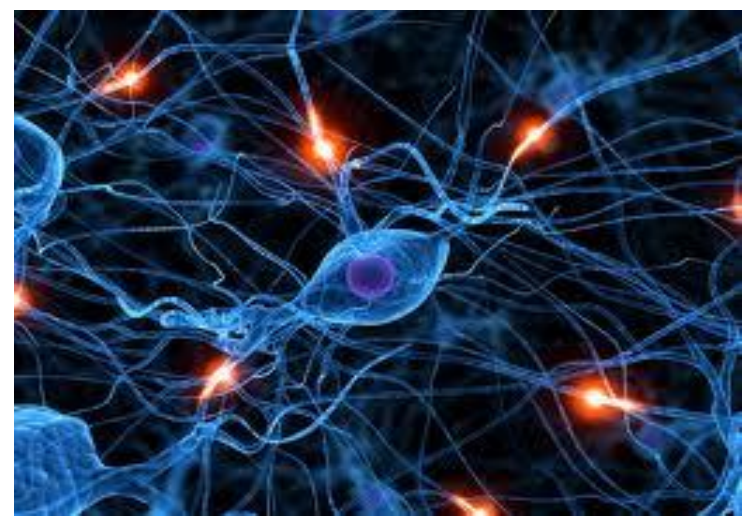

Fig.1. Neural Networks

The neural networks exhibit mapping capability, they can map input pattern to their associated output patterns. They can learn by examples. Neural network architectures can be trained with known examples of a problem before they are tested for their inference capacity on unknown instances of the problem. They can identify new objects previously untrained. They possess the capacity to generalize. Thus, they can predict new outcomes from past trends. They are robust systems and fault tolerant. They can recall full patterns from incomplete, partial or noisy patterns. They can also process information in parallel, at high speed and in a distributed manner.

\section{ARCHITECTURE OF NEURAL NETWORKS 1. Feed-forward networks:}

Feed-forward ANNs allow signals to travel one way only; from input to output. There is no feedback (loops) i.e. the output of any layer does not affect that same layer. Feed-forward ANNs tend to be straight forward networks that associate inputs with outputs. They are extensively used in pattern recognition. This type of organization is also referred to as bottom-up or top-down.

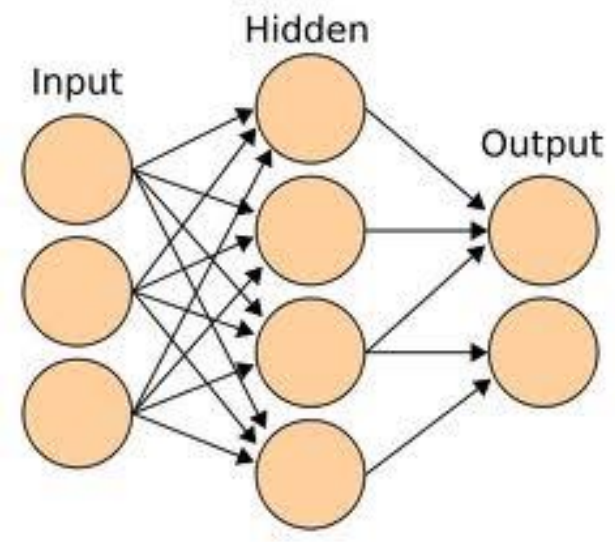

Fig.2 Feed-Forward Networks 


\section{Feedback networks:}

Feedback networks can have signals travelling in both directions by introducing loops in the network. Feedback networks are very powerful and can get extremely complicated. Feedback networks are dynamic; their 'state' is changing continuously until they reach an equilibrium point. They remain at the equilibrium point until the input changes and a new equilibrium needs to be found. Feedback architectures are also referred to as interactive or recurrent, although the latter term is often used to denote feedback connections in single-layer organizations.

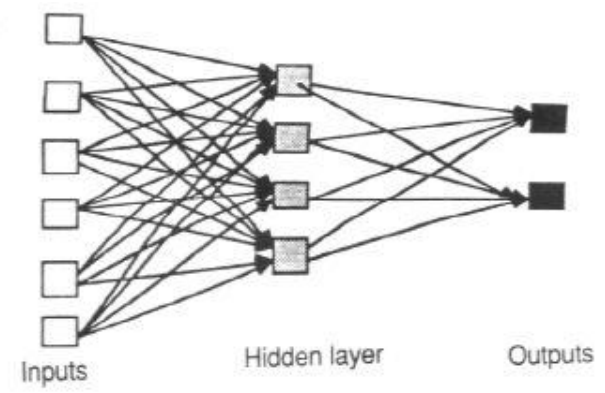

Fig.3 Feedback Networks

\section{NEURAL NETWORKS LAYER}

The common type of artificial neural network consists of three groups, or layers, of units: a layer of "input" units is connected to a layer of "hidden" units, which is connected to a layer of "output" units. The activity of the input units represents the raw information that is fed into the network. The activity of each hidden unit is determined by the activities of the input units and the weights on the connections between the input and the hidden units. The behavior of the output units depends on the activity of the hidden units and the weights between the hidden and output units.

This simple type of network is interesting because the hidden units are free to construct their own representations of the input. The weights between the input and hidden units determine when each hidden unit is active, and so by modifying these weights, a hidden unit can choose what it represents. We can also distinguish single-layer and multilayer architectures. The single-layer organization, in which all units are connected to one another, constitutes the most general case and is of more potential computational power than hierarchically structured multi-layer organizations. In multi-layer networks, units are often numbered by layer, instead of following a global numbering.

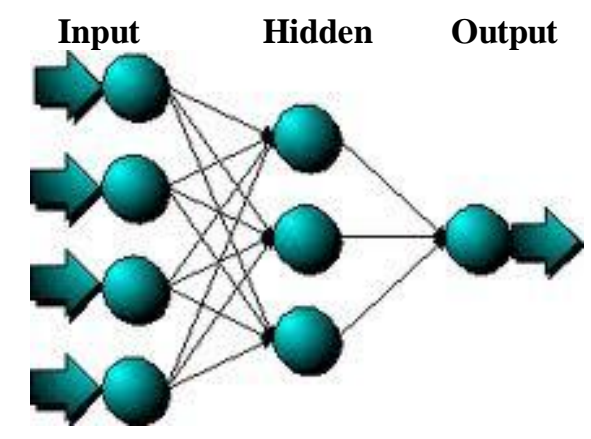

Fig.4 Neural Networks Layer

\section{APPLICATIONS OF NEURAL NETWORKS}

Neural Networks have broad applications to the real world business problems. They have already been successfully applied in many industries. Since neural networks are best at identifying patterns or trends in data, they are well suited for prediction or forecasting. These include Sales forecasting, Industrial process control, Customer research, Data validation, Risk management and Target marketing.

\section{BUSINESS}

Human beings are continuously engaged in some activity or other in order to satisfy their unlimited wants. Every day we come across the word 'business' or 'businessman' directly or indirectly. Business has become essential part of modern world.

Business is an economic activity, which is related with continuous and regular production and distribution of goods and services for satisfying human wants.

All of us need food, clothing and shelter. We also have many other household requirements to be satisfied in our daily lives. We also have many other household requirements to be satisfied in our daily lives. We met these requirements from the shopkeeper. The shopkeeper gets from wholesalers. The wholesalers get from manufactures. The shopkeepers, the wholesalers and the manufactures are doing business and therefore they are called as Businessman.

\section{TYPES OF BUSINESS}

1. Agriculture and Mining businesses are concerned with the production of raw material, such as plants or minerals.

2. Financial businesses include banks and other companies that generate profit through investment and management of capital.

3. Information businesses generate profits primarily from the resale of intellectual property and include movies, studios, publishers and packaged software companies.

4. Manufacturers produce products, from raw materials or component parts, which they then sell at a profit. 
Companies that make physical goods, such as cars or pipes, are considered manufacturers.

5. Real estate businesses generate profit from the selling, renting, and development of properties comprising land, residential homes, and other kinds of buildings.

6. Retailers and distributors act as middle-men in getting goods produced by manufacturers to the intended consumer, generating a profit as a result of providing sales or distribution services. Most consumer-oriented stores and catalog companies are distributors or retailers.

7. Service businesses offer intangible goods or services and typically generate a profit by charging for labor or other services provided to government, other businesses, or consumers. Organizations ranging from house decorators to consulting firms, restaurants, and even entertainers are types of service businesses.

8. Transportation businesses deliver goods and individuals from location to location, generating a profit on the transportation costs.

9. Utilities produce public services such as electricity or sewage treatment, usually under a government charter.

Indian economy is one of the fastest growing in the world. It has seen an explosion in the increase of foreign business investment, outsourcing and Indian companies venturing overseas. Doing business in India offers immense benefits for international organizations, however there are a number of key cultural challenges that can create misunderstanding and conflict as well as huge direct and indirect costs to the organization if overlooked.

\section{BUSINESS FORECASTING:}

Business forecasting is a process used to estimate or predict future patterns using business data. Some examples of business forecasting include estimating quarterly sales, product demand, customer lifetime value and churn potential, inventory and supply-chain reorder timing, workforce attention, website traffic, and predicting exposure to fraud and risk.

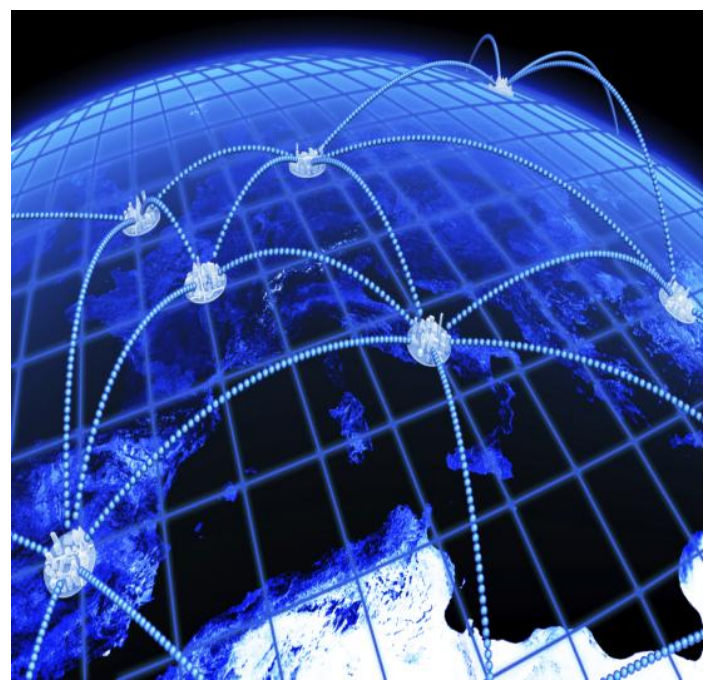

Fig.5 Business Enhancement

Several powerful estimation functions are commonly used to perform business forecasting: time series analysis, causal models, and regression analysis. Business forecasting supports executives, analysts and end users in decision-making using decision support systems such as business intelligence.

\section{FACTORS INFLUENCING BUSINESS FORECASTING}

The factors which influence business forecasting are:

1. Preparation of Budget- Decision-making involves budget allocation i.e., resource allocation to various aspect of decision. Budget may be allocated to various factors of production.

2. Future Development- Strategic plans are usually expected to have a significant future prosperity of the organization. This is because there is a long-term commitment. In case of absence of long-term commitment the firm cannot achieve future development.

3. Orientation- Strategic planning should keep in view of the competition existing in the market. Sometimes firms have to face non-price competition.

4. Factors of Environment- Plans are always influenced by business environment always influencing factor for decision-making. There are external or internal factors that influence business. Buyers, Suppliers, government and competitors are likely to react in accordance with changes in environment. Thus business also should act in the same passion. 
5. Risk- Strategic plans mostly face the problem of risk. The plans should able to tackle the risk bearing capacity. Risk and uncertainty are two important aspects, which cannot be expected by business man.

\section{TRAINING THE NEURAL NETWORKS}

Once a network has been structured for a particular application, that network is ready to be trained. To start this process the initial weights are chosen randomly. Then, the training, or learning, begins. The ANN has been trained by exposing it to sets of existing data (based on the follow up history of cancer patients) where the outcome is known. Multi-layer networks use a variety of learning techniques; the most popular is back - propagation algorithm. It is one of the most effective approaches to machine learning algorithm. Information flows from the direction of the input layer towards the output layer. A network is trained rather than programmed. Learning in ANN's is typically accomplished using examples. This is also called 'training' in ANN's because the learning is achieved by adjusting the connection weights in ANN's iteratively so that trained (or learned).The number of iterations of the training algorithm and the convergence time will vary depending on the weight Initialization. After repeating this process for a sufficiently large number of training cycles the network will usually converge to some state where the error of the calculations is small. In this case one says that the network has learned a certain target function. Learning techniques are often divided into supervised, unsupervised and reinforcement learning.

It is well known that the performance of learning systems on the training data often does not reflect the performance on unknown data. This is due to the fact that the system often adapts well on training to the particularities of the training data. Therefore, the training data should be randomly chosen from all available data. It should represent the typical data properties. If we have initially a bias in the training data, we will encounter performance problems for the test data later. In order to test the real generalization abilities of a network to unknown data, it must be tested by classified, but yet unknown data, the test data that should not contain samples coming from patients of the training data. We have to face the fact that patient data is very individual and it is difficult to generalize from one patient to another. Ignoring this fact would pretend better results than a real system could practically achieve.

\section{NEURAL NETWORKS BUSINESS FORECASTING}

\section{Neural Networks towards Marketing Management:}

There is a marketing application which has been integrated with a neural network system. The Airline Marketing Tactician (a trademark abbreviated as AMT) is a computer system made of various intelligent technologies including expert systems. A feed forward neural network is integrated with the AMT and was trained using backpropagation to assist the marketing control of airline seat allocations. The adaptive neural approach was amenable to rule expression. Additionally, the application's environment changed rapidly and constantly, which required a continuously adaptive solution. The system is used to monitor and recommend booking advice for each departure. Such information has a direct impact on the profitability of an airline and can provide a technological advantage for users of the system.

While it is significant that neural networks have been applied to this problem, it is also important to see that this intelligent technology can be integrated with expert systems and other approaches to make a functional system. Neural networks were used to discover the influence of undefined interactions by the various variables. While these interactions were not defined, they were used by the neural system to develop useful conclusions. It is also noteworthy to see that neural networks can influence the bottom line.

Neural networks change the way to use information in marketing. With such a new information technology, a company using a neural network, will eventually have affordable, near real-time access to all the raw numbers it wants. These data may be obtained from consumer credit card applications, point-of -purchase credit-card sales, and credit agency reports. The real difference among competitors will be the quality of analysis each performs and the capacity of decisions flowing from it. Neural networks help managers gather and process information, such as age, income, credit history, and products purchased.

Neural networks have been applied to a wide range of information-processing activities, such as associate memory, pattern classification and clustering, and function approximation and prediction. These applications are characterized by unstructured decision processes, multiobjectives and multiple stage decision activities. Such applications may not be efficiently supported using expert and decision support systems technologies. 
IOSR Journal of Engineering

Apr. 2012, Vol. 2(4) pp: 831-836

\section{Neural Networks towards Financial Management:}

Business is a diverted field with several general areas of specialization such as accounting or financial analysis. Almost any neural network application would fit into one business area or financial analysis. There is some potential for using neural networks for business purposes, including resource allocation and scheduling. There is also a strong potential for using neural networks for database mining that is, searching for patterns implicit within the explicitly stored information in databases. Most of the funded work in this area is classified as proprietary. Thus, it is not possible to report on the full extent of the work going on. Most work apply neural networks, such as the Hopfield-Tank network for optimization and scheduling.

Neural networks are revolutionizing virtually every aspect of financial \& investment decision-making. Financial firms worldwide are using neural networks to forecast markets, analyze credit risks, \& improve backoffice operations.

From the range of AI techniques, the one that deals best with uncertainty is the Artificial Neural Network (ANN). Dealing with

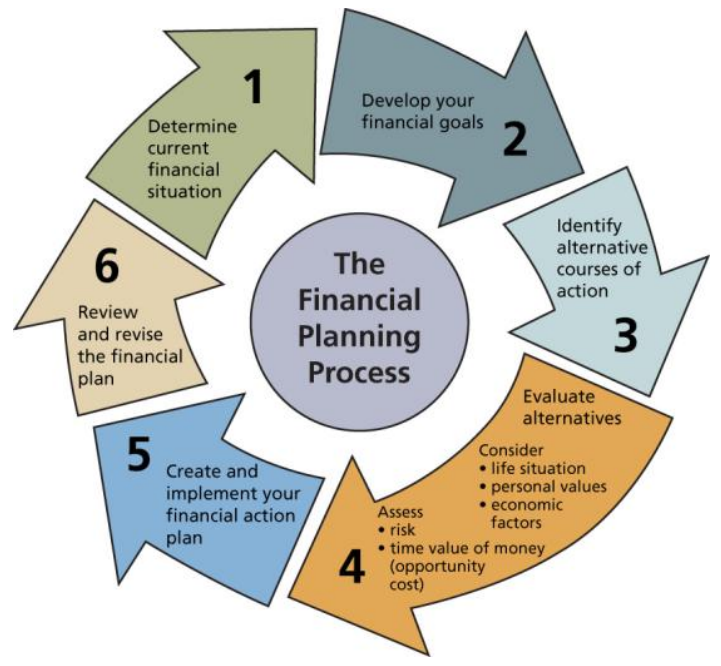

Fig.6 Financial Planning Process

uncertainty in finance primarily involves recognition of patterns in data and using these patterns to predict future events. Accurate prediction of economic events, such as interest rate changes and currency movements currently ranks as one of the most difficult exercises in finance; it also ranks as one of the most critical for financial survival. ANNs handle these problems better than other AI techniques because they deal well with large noisy data sets. Unlike expert systems, however, ANNs are not transparent, thus making them difficult to interpret. The Neural Networks will evaluate the following financial activities.
$>$ Credit authorization screening

$>$ Mortgage risk assessment

$>$ Project management and bidding strategy

$>$ Financial and economic forecasting

$>$ Risk rating of exchange-traded, fixed income investments.

$>$ Detection of regularities in security price movements

$>$ Prediction of default and bankruptcy

\section{Neural Network towards Operation Management:}

There are many areas of operation management, particularly scheduling and planning, where neural networks have been used successfully. The scheduling of machinery, assembly lines and cellular manufacturing using neural networks have been popular research topics over the last decade. Other scheduling problems like timetabling, project scheduling and multiprocessor task scheduling have also been successfully attempted. All of these approaches are based upon the Hopfield Neural Network and the realization of Hopfield and Tank that these networks could solve complex optimization problems. Recently, alternative neural network approaches like neuro-dynamic programming have also been used to solve related problems. The other area of operations management which benefits from neural networks is quality control. Neural Networks can be integrated with traditional statistical control techniques to enhance their performance.

\section{Neural Network towards Risk Management:}

Neural Systems makes use of a supervised network to mimic the recommendations of money managers on the optimal allocation of assets among Treasury instruments. The application demonstrated how well an ANN can be trained to recognize the shape and evolution of the interest-yield curve and to make recommendations as to long or short positions in the US Treasury market.

The network was trained on measured and calculated economic indicators, such as the evolution of interest rates, price changes, and the shape and speed of the change of the yield curves. The network could then determine the optimal allocation among segments in various Treasury instruments being measured against a benchmark or comparator performance index. It could determine also the dynamic relationship between different variables in portfolio management and risk control. Consequently, it allowed more active control of portfolio's level of certainty. Based on the experience gained with this application, another ANN with a higher level of complexity was subsequently developed. 


\section{CONCLUSIONS \& FURTHER ENHANCEMENT:}

1. Neural Network is defined as the ability of a group to solve more problems than its individual members.

2. The idea brings that a group of people can solve problems efficiently and offer greater insight and a better answer than any one individual could provide.

3. The applications of Neural Network enhance an innovative business model for an enterprise.

4. Role of Neural Network in an enterprise brings effectiveness.

5. Further work will be carried out towards the Mathematical modeling of neural networks and various parameters will be engaged so as to get the required result to desired degree of accuracy.

\section{REFERENCES}

\section{Journal Papers:}

[1] Neural Networks at Pacific Northwest National Laboratory

http://www.emsl.pnl.gov:2080/docs/cie/neural/neural.h omepage.html

[2] Artificial Neural Networks in Business http://www.emsl.pnl.gov:2080/docs/cie/techbrief/NN.t echbrief.html

[3] The Problem of Neural Networks in Business Forecasting: An Attempt to Reproduce the Hill, O'Connor and Remus Study.

[4] Neural networks in business: a survey of applications (1992-1998) A Vellido, P.J.G Lisboa, J Vaughan

[5] Forecasting with artificial Neural Networks:: The state of the art.

[6] D Van Heemst, P M Den Reijer, R G J Westendorp in European journal of cancer Oxford England 1990 (2007)

[7] Time series forecasting using neural networks. Michael Nelson, Tim Hill, William Remus, Marcus O'Connor'

\section{Books:}

[8] An introduction to neural computing. Aleksander, I. and Morton, H. $2^{\text {nd }}$ edition

[8] Neural Networks by Eric Davalo and Patrick Naim $2^{\text {nd }}$ edition

[9] Learning internal representations by error propagation by Rumelhart, Hinton and Williams (1986). $1^{\text {st }}$ edition

[10]An Introduction to Neural Networks by Ben Krose, Patrick van der Smagt, 1996

[11]Neural Networks in Hardware: Architectures, Products and Applications by Clark S. Lindsey, 1988

\section{Theses:}

[11] Industrial Applications of Neural Networks (research reports Esprit, I.F.Croall, J.P.Mason)

[12] Klimasauskas, CC. (1989). The 1989 Neuro Computing Bibliography. Hammerstrom, D. (1986). A Connectionist/Neural Network Bibliography.

[13] DARPA Neural Network Study (October, 1987February, 1989). MIT Lincoln Lab. Neural Networks, Eric Davalo, Patrick Naim Assimov, I (1984, 1950), Robot, Ballatine, New York.

[14] Studies in Artificial Neural Network Modeling by Ninan Sajeeth Philip, Cochin University of Science and Technology.

[15] Neural Networks for Variation Problems in Engineering by Dr. Eva Balsa Canto, Technical University of Catalonia. 